\title{
Galectin-9 Is a Novel Regulator of Epithelial Restitution
}

Brian S. Robinson, ${ }^{*}$ Bejan Saeedi, ${ }^{*}$ Connie M. Arthur ${ }^{\dagger}$ Josh Owens, ${ }^{\ddagger}$ Crystal Naudin,${ }^{\ddagger}$ Nourine Ahmed, ${ }^{\dagger}$ Liping Luo, ${ }^{\ddagger}$ Rheinallt Jones, ${ }^{\ddagger}$ Andrew Neish, ${ }^{*}$ and Sean R. Stowell ${ }^{* \dagger}$

From the Departments of Pathology and Laboratory Medicine, ${ }^{*}$ and Pediatrics, ${ }^{\ddagger}$ and the Center for Transfusion and Cellular Therapies, ${ }^{\dagger}$ Emory University School of Medicine, Atlanta, Georgia

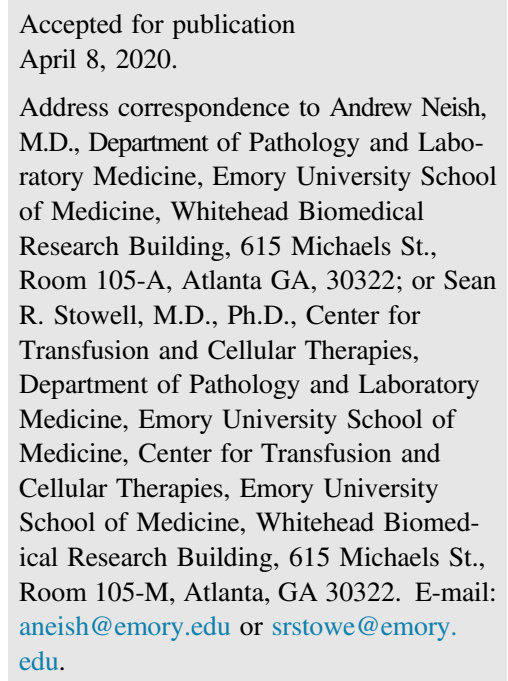

Increasingly, the ß-galactoside binding lectins, termed galectins, are being recognized as critical regulators of cell function and organismal homeostasis. Within the context of the mucosal surface, galectins are established regulators of innate and adaptive immune responses, microbial populations, and several critical epithelial functions, including cell migration, proliferation, and response to injury. However, given their complex tissue distribution and expression patterns, their role within specific processes remains poorly understood. We took a genetic approach to understand the role of endogenous galectin-9 (Gal9), a mucosal galectin that has been linked to inflammatory bowel disease, within the context

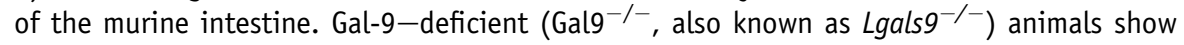
increased sensitivity to chemically induced colitis and impaired proliferation in the setting of acute injury. Moreover, Gal9 $9^{-/-}$-derived enteroids showed impaired growth ex vivo. Consistent with a model in which endogenous Gal-9 controls epithelial growth and repair, Gal9-/animals showed increased sensitivity to intestinal challenge in multiple models of epithelial injury, including acute irradiation injury and ectopic wound biopsies. Finally, regenerating crypts from patient biopsies showed increased expression of Gal-9, indicating these processes may be conserved in humans. Taken together, these studies implicate Gal- 9 in the regulation of cellular proliferation and epithelial restitution after intestinal epithelial injury. (Am J Pathol 2020, 190: 1657-1666; https://doi.org/10.1016/j.ajpath.2020.04.010)
The epithelial lining of the gastrointestinal tract physically separates luminal contents from underlying tissue compartments and serves as an essential barrier against pathogens and exogenous stress. Defects in epithelial barrier function and/or epithelial restitution programs after injury can drive altered tissue homeostasis and can result in increased morbidity and mortality. ${ }^{1,2}$ Not surprisingly, defects in epithelial repair programs have been described in a number of disease states that impact gastrointestinal tract function, including inflammatory bowel disease. ${ }^{3,4}$ Although much is known regarding the intracellular signaling pathways that drive epithelial restitution, less is understood regarding the secreted factors that drive repair in the face of injury and how they orchestrate tissue restitution and homeostasis.

Galectins are a class of secreted lectins that have garnered significant attention in recent years because of their ability to regulate diverse sets of processes critical to epithelial biology, including cell-cell adhesion, cell migration, lipid raft formation, cell-cycle progression, and apoptosis. ${ }^{5}$ Originally isolated from the electric organ of the electric eel, up to 15 galectins have been described in vertebrates that all are unified by their ability to bind terminal $\beta$-galactosides on cognate glycolipids and glycoproteins via a conserved approximately 130 amino acid carbohydrate recognition domain (CRD). ${ }^{6-9}$ Galectins are categorized broadly into 3 classes, including prototypic galectins (Gal-1, -2, -5, -7, -10, -11, -13, and -14) containing a single CRD; tandem-repeat galectins (Gal-4, -6, -8, -9, and

Supported by a Burroughs Wellcome Trust Career Award for Medical Scientists, and NIH grants DP5OD019892 (S.R.S.), U01CA242109 (S.R.S.), R01 AI064462 (A.S.N.), and T32DK108735 (B.S.R.).

Disclosures: None declared. 
-12), which contain two CRDs separated by a linker region, and chimeric galectins (Gal-3), which contain a single CRD with a long N-terminal tail. ${ }^{10-12}$ Within the context of the mucosal surface, previous results have shown that galectin expression is limited to galectins- $1,-3,-4,-7,-8$, and $-9 .{ }^{13}$ Genetic and biochemical studies in models of colitis have shown that Gal-3-deficient $\left(\mathrm{Gal}^{-} 3^{-1-}\right.$, also known as Lgals $^{-1-}$ ) mice show increased disease severity, whereas intraperitoneal injection of Gal-1, Gal-4, or Gal-9 can attenuate colitis in the setting of similar colitis models. ${ }^{14-20}$ These results suggest a role for galectins in epithelial function and repair. Consistent with this, altered expression of galectins has been linked to patients with inflammatory bowel disease and/or active colitis. ${ }^{21}$ However, the role of galectins in the restitution of epithelia after injury remains incompletely understood.

To examine the impact of galectins on epithelial injury repair in more detail, we examined the role of endogenous Gal-9, a mucosal galectin that has been linked to 1 of nearly 30 loci associated with inflammatory bowel disease in genome-wide association studies, ${ }^{22}$ in the regulation of epithelial responses to injury. The study results show that Gal-9 ${ }^{-/-}$(also known as Lgals $9^{-/-}$) mice are more sensitive than wild-type (WT) mice to intestinal injury in multiple models of epithelial injury, including chemical colitis, acute irradiation injury, and in vivo wound biopsies. Consistent with an intrinsic role of galectin-9 in epithelial repair, crypts isolated from $\mathrm{Gal}_{-} 9^{-/-}$mice show impaired growth in ex vivo cultures. Impaired restitution observed in Gal-9 $9^{-1-}$ mice is linked to defective proliferative responses in the epithelia of $\mathrm{Gal}-9^{-/-}$mice. Finally, Gal-9 expression is induced at the proliferating edges of human biopsies. These collective data indicate that Gal-9 is a key regulator of epithelial proliferation and intestinal mucosal restitution.

\section{Materials and Methods}

\section{Mouse Models}

WT C57BL/6 mice were purchased from Jackson Laboratories (Bar Harbor, ME). Gal- $9^{-1-}$ on a B6 background were obtained from the Consortium for Functional Glycomics (La Jolla, CA) as a kind gift from Stephen M. Berkeley. All animal experiments were approved by the Institutional Animal Care and Use Committee at Emory University and were performed according to NIH guidelines. Animals were maintained on a 12-hour light/dark cycle and had ad libitum access to a standard diet and water using similar protocols as performed previously. ${ }^{23-25} \mathrm{Ex}-$ periments were performed on mature animals (age, 8 to 12 wk) in both male and female mice. Histologic and endoscopic analyses were performed in a blinded fashion (B.S.R. and A.N.), and the sample sizes represent biological replicates. Genotyping was performed by PCR using established protocols provided by the Center of Functional Glycomics.

\section{Dextran Sodium Sulfate Colitis}

Mice were allowed free access to food and drinking water containing 3\% (wt/vol) dextran sodium sulfate (DSS; molecular mass, 36 to $50 \mathrm{kDa}$; MP Biomedicals, Irvine, CA) for 9 days. Daily clinical assessment of DSS-treated animals included evaluation of stool consistency, detection of blood in stool, and body weight loss measurements. ${ }^{26}$ An individual score (range, 0 to 4 ) was attributed to each one of these parameters, and a disease activity index ranging from 0 to 12 was calculated by combining all three scores. An analysis of the percentage of ulceration in whole colon samples was performed using scanned photomicrographs of hematoxylin and eosin-stained histologic sections of the whole colon (Swissroll) of the mice and measuring the ulcerated (denuded mucosa) area to compare it with the colon length of each mouse.

\section{Acute Irradiation}

For irradiation experiments, mice whole bodies were exposed to 8 Gy of $\gamma$-radiation using a $\gamma$-Cell $40{ }^{137} \mathrm{Cs}$ irradiator (RS-2000 Series; Rad Source Technologies Inc, Buford, GA) at a dose rate of $75 \mathrm{rad} /$ minute. ${ }^{27}$ Body weights and mortality were monitored twice daily. Histologic sections of the colon were prepared from five irradiated animals per treatment performed as previously described. ${ }^{28}$

\section{Histologic Assessment}

For colitis experiments, hematoxylin and eosin-stained slides of colonic tissue was scored for the degree of colitis using an established 2-tiered system that evaluates both the degree of inflammation and the extent of epithelial injury. ${ }^{29}$ Sections will be scored in a blinded fashion by a board-certified pathologist (B.S.R.). For crypt height assessments in colitis experiments, $10 \times$ objective photomicrographs were taken of intact epithelium immediately adjacent to ulcerated foci, and crypt length was measured using the length tool on FIJI software version $2.0 .0-\mathrm{rc}-69 / 1.52 \mathrm{i}^{30}$

\section{Immunofluorescence}

Immunofluorescence was performed on formalin-fixed, paraffin-embedded samples using standard heat-induced epitope retrieval in citrate buffer with a $\mathrm{pH}$ of 6. Samples then were blocked with $3 \% \mathrm{wt} / \mathrm{vol}$ bovine serum albumin in phosphate-buffered saline for 1 hour and incubated with primary antibody overnight at $4^{\circ} \mathrm{C}$, washed, and incubated for 1 hour with fluorophore-labeled secondary antibodies, then mounted in p-phenylene. Incubations were performed at room temperature, and between incubations, sections were washed with Tris-buffered saline. Images were taken on a LSM 510 confocal microscope (Zeiss, Oberkochen, Germany) with Plan-NEOFLUAR $\times 100 / 1.3$ oil, $\times 40 / 1.3$ oil, and $\times 20 /$ 0.5 dry objectives, with software supplied by the vendor. Primary antibodies included allophycocyanin-conjugated 
anti-mouse Ki-67 (cat. 652405, 1:500; BioLegend, San Diego, CA), $\beta$-catenin (\#8480, 1:100; Cell Signaling, Danvers, MA), and Gal-9 (\#54330, 1:100; Cell Signaling). ${ }^{31}$

\section{Ki-67 Proliferation Index}

Fresh-frozen sections were immunostained with Ki-67, DAPI, and B-catenin, and imaged on an LSM 510 confocal microscope (Zeiss) with Plan-NEOFLUAR $\times 20 / 0.5$ dry objectives. For each section, ulcerated edges were identified and imaged, and the percentage of Ki-67-positive nuclei per crypts adjacent to ulcerated edges was determined via manual quantification using the Cell Counter tool on the FIJI software system. All images we obtained under identical optical settings.

\section{Transcriptional Analysis}

For transcriptional analysis, whole colon was dissected and snap-frozen in liquid nitrogen. Colonic tissue then was homogenized using a liquid nitrogen-cooled mortar and pestle. Colonic tissue $(50 \mathrm{mg}$ ) was disrupted mechanically in TRIzol (Invitrogen, Carlsbad, CA) using a MagnaLyser with MagnaLyser beads (Roche, Basel, Switzerland). RNA was prepared according to the TRIzol manufacturer's instructions. RT-PCR was performed using SybrGreen supermix (Bio-Rad, Hercules, $\mathrm{CA})$ using the following primers: murine Actin forward: $5^{\prime}$ ACCTTCTACAATGAGCTGCG-3', murine Actin reverse: 5'-CTGGATGGCTACGTACATGG-3', murine Gal9 forward: 5'-GGATGCCCTTTGAGCTTTGC-3', murine Gal9 reverse: 5'-GGGCAGGACGAAAGTTCTGA-3'. Data were analyzed using the $2^{\wedge} \Delta \Delta \mathrm{Ct}$ method. ${ }^{32}$

\section{Organoid Culture}

The organoid ex vivo culture method was adapted from Sato and colleagues ${ }^{33,34}$ using previously described modifications. ${ }^{28,35}$ Briefly, isolated mouse intestines (approximately $15 \mathrm{~cm}$ of jejunum) were washed 15 times with cold phosphate-buffered saline without $\mathrm{Ca}^{2+} / \mathrm{Mg}^{2+}$, cut into approximately 3- to 5-mm pieces, and incubated in Gentle Cell Dissociation Reagent (Stem Cell Technologies, Vancouver, Canada). After removal of the Gentle Cell Dissociation Reagent and resuspension with phosphate-buffered saline with $0.1 \%$ bovine serum albumin, the villi and mucus were removed with a 70- $\mu \mathrm{m}$ cell strainer (BD Biosciences, Franklin Lakes, NJ). Isolated crypts were then centrifuged at $4^{\circ} \mathrm{C}$ at $290 \times g$ for 5 minutes and resuspended in cold Dulbecco's modified Eagle's medium/F12 with $15 \mathrm{mmol} / \mathrm{L}$ HEPES. One hundred fifty intestinal crypts were plated into a dome containing a crypt:Matrigel ratio of 1:1 (356255; Invitrogen). Enteroid culture media included murine IntestiCult Organoid Growth Medium (Stem Cell Technologies, Vancouver, Canada) supplemented with $100 \mathrm{IU}$ of penicillin streptomycin. Passage of enteroids derived from single cells was performed every 7 to 10 days. Briefly, enteroid passage included washing cultures with cold sterile phosphate-buffered saline, dissociation of enteroids for 15 minutes in Gentle Cell Dissociation Reagent, and seeding in Matrigel performed at a concentration of 150 crypts per dome. Light microscopy was performed on cultures 9 days after passaging, with representative images selected after counting 6 images at $100 \times$ magnification from each group ( $n=6$ cultures per group). Spheroids were defined as enclosed three-dimensional structures without buds, whereas enteroids were defined as budding spheres having one or more bud from the main organoid structure.

\section{Wound Biopsy}

To generate discrete mucosal injuries in the mouse colon and to monitor their recovery, a high-resolution miniaturized colonoscope system (Coloview Veterinary Endoscope; Karl Storz, Tuttlingen, Germany) was used as described previously. ${ }^{36}$ This system consisted of a miniature rigid endoscope (outer diameter, $1.9 \mathrm{~mm}$ ); a xenon light source; a triple-chip, high-resolution, charge-coupled device camera; and an operating sheath with instrument channels and an air/ water injection bulb to regulate inflation of the mouse colon (all from Karl Storz). Briefly, mice were anesthetized using ketamine and xylazine and the endoscope with an outer operating sheath was inserted into the mid-descending colon and the mucosa was surveyed to the anorectal junction. Using flexible biopsy forceps (diameter, F3), a single fullthickness areas of the entire mucosa and submucosa was removed. On a flat-panel color monitor, endoscopic procedures were viewed with high-resolution images $(1024 \times 768$ pixels $)$. A $0.5-\mathrm{mm}$ rod was used to calibrate measurements of wound size according to the methods of Seno et al. ${ }^{37}$ ImageJ software version 1.50i (NIH, Bethesda, MD; https://imagej.nih.gov/ij) was used to analyze the wound sizes. The percentage of wound closure was determined as the change in wounds size from day 3 (72 hours after biopsy) relative to day 1 ( 24 hours after biopsy).

\section{Statistics}

Statistical analysis was performed using Prism software version 7 (GraphPad Software, San Diego, CA) and 1-way analysis of variance was performed with the Tukey post-test or the $t$-test. Significance was determined by $P<0.05$.

\section{Results}

\section{Gal-9-1- Mice Are Sensitive to Chemically Induced Colitis}

Genome-wide association studies have indicated a link between Gal-9 and inflammatory bowel diseases, ${ }^{22}$ yet its role in the development of colitis remains untested. This study analyzed the expression of Gal-9 in mice exposed to DSS, which induced chemical colitis in mice, and found that Gal9 was increased significantly after DSS treatment. To test 

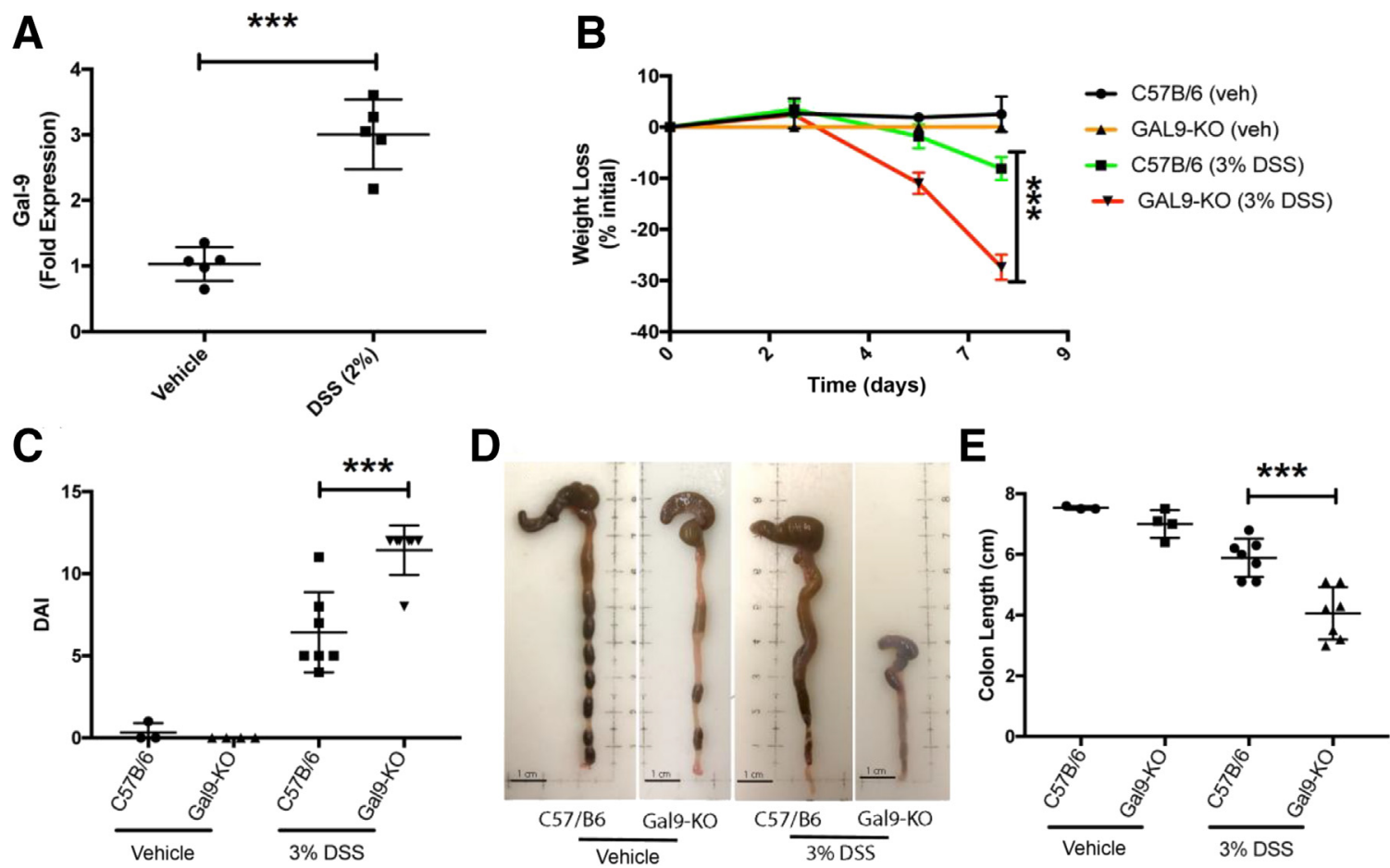

Figure 1 Dextran sodium sulfate (DSS)-induced colitis in wild-type (WT) and galectin-9-deficient (Gal9 ${ }^{-/-}$also known as $L g a l s 9^{-/-}$) mice. A: Gal-9 expression in colon preparations from vehicle-treated and DSS-treated mice (treated with 2\% DSS for 6 days). B and C: C57BL/6 (WT) and Gal-9 ${ }^{-1-}$ mice were administered either vehicle (veh) or $3 \%$ DSS and monitored for weight loss (B) and disease activity (C). D and E: After 8 days of treatment, mice were sacrificed, colons were dissected (D), and colon length was quantified (E). $n=5$ per group (A). ${ }^{* *} P<0.0005$. Scale bars $=1 \mathrm{~cm}(\mathbf{D})$. DAI, disease activity index; K0, knockout.

the endogenous requirement of Gal-9 in colitis, WT (C57BL/6) or Gal-9 ${ }^{-l-}$ mice were challenged with $3 \%$ dextran sodium sulfate and monitored their response to injury. Gal- $9^{-1-}$ animals showed significantly more weight loss starting at approximately day 6 compared with their WT C57BL/6 controls (Figure 1A). Moreover, when analyzed for disease activity, Gal-9 $9^{-1-}$ animals showed significantly more disease activity than the similarly challenged WT controls (Figure 1B). After 8 days of colitis, animals were sacrificed for histologic assessment. Although vehicle-treated $\mathrm{Gal}-9^{-1-}$ and WT colon lengths remained the same, DSS-treated colons were significantly shorter than DSS-treated WT colons, consistent with increased disease activity (Figure 1, C-E). Histologic analysis showed that although vehicle-treated WT and Gal- $9^{-1-}$ colons remained unremarkable (Figure 2, A and B), DSS-treated samples showed marked ulceration with mixed acute and chronic inflammation (Figure 2, C and D). In blinded fashion (B.S.R. and A.N.), samples were scored for extent of mucosal injury and severity of inflammation using wellestablished criteria. ${ }^{29}$ Although the total histologic activity was not significantly different between groups (Figure 2E), Gal- $9^{-1-}$ animals showed significantly increased indices in epithelial injury compared with their WT counterparts (Figure 2G), with slightly decreased indices of inflammation (Figure 2F). In light of the increased epithelial injury, the percentage of epithelia lost per sample was compared between DSS-treated WT and Gal-9 ${ }^{-/-}$animals. Consistent with more extensive epithelial injury, samples derived from Gal- $9^{-1-}$ DSS-treated animals showed significantly more epithelial loss than their WT counterparts (Figure 2H). Taken together, these findings show that animals deficient in Gal-9 expression are more sensitive to chemically induced colitis, with an extensive loss in epithelia in treated animals.

\section{Gal9 ${ }^{-/}$Mice Show Altered Proliferation at Wound Edges}

The disproportionate epithelial loss in DSS-treated Gal- $9^{-1-}$ animals indicated a possible defect in Gal-9-deficient epithelia. To test this, the proliferative response in WT and Gal- $9^{-1-}$ animals were examined. Examination of the ulcer edges in Gal- $9^{-\prime}$ animals showed significantly decreased crypt length in epithelia adjacent to ulcer edges (Figure 3C), with the crypts of WT ulcer edges appearing more hyperchromatic and also possessing increased mitotic figures (Figure 3, A and B). To examine the proliferative capacity of adjacent crypts, tissue sections were stained with $\mathrm{Ki}-67 / \mathrm{MiB}$ 1 , an epitope that labels cells in G0/G1 that is increased in mitotically active cells. ${ }^{38}$ Consistent with a model in which Gal- $9^{-/-}$animals have defective restitutive responses, Ki-67 labeling was reduced significantly on ulcer edges compared with WT animals (Figure 3, D-F) (Ki-67 proliferation index 

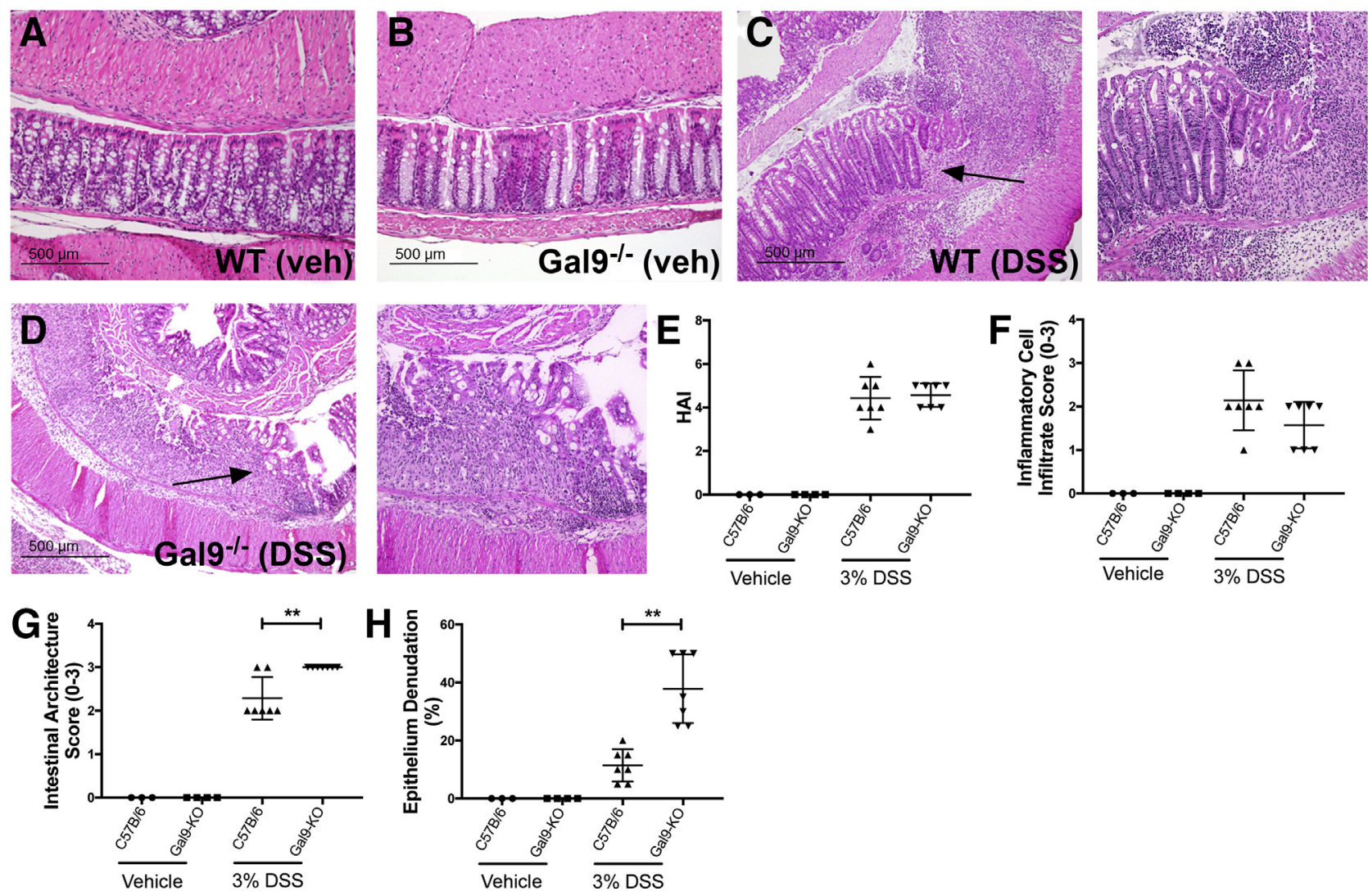

Figure 2 Histologic analysis of dextran sodium sulfate (DSS)-treated wild-type (WT) and galectin-9-deficient (Gal-9 ${ }^{-/-}$also known as $L g a l s 9^{-/-}$) mice. A-D: Representative photomicrographs of hematoxylin and eosin-stained sections from the colonic mucosa obtained from day 8 preparations of either vehicle-treated WT and Gal- $9^{-1-}$ mice (A and B, respectively) or DSS-treated WT and Gal- $9^{-1-}$ mice (C and $\mathbf{D}$, respectively). Arrows indicate crypts at ulcerated edge. Higher-magnification photomicrographs (20x objective) are shown on the right (C and $\mathbf{D})$ ). E-G: Hematoxylin and eosin-stained sections were scored for the degree of colitis as determined by the histologic activity index $(\mathbf{E})$, which is a composite of the extent of inflammatory infiltrate (F) and the degree of epithelial injury (G). H: The percentage of epithelial loss as calculated based on the amount of intact epithelia. ${ }^{* *} P<0.005$. Scale bars $=500 \mu \mathrm{m}(\mathbf{A}-\mathbf{D})$. HAI, histology activity index; K0, knockout; veh, vehicle.

of $29.6 \% \pm 2.6 \%$ in WT compared with $9.6 \% \pm 2.8 \%$ in Gal- $9^{-1-}$ animals; $\left.P<0.005\right)$.

\section{Gal- $9^{-/-}$-Derived Epithelial Organoids Show Defective Growth Ex Vivo}

The above data indicate that epithelia deficient in Gal-9 have impaired responses to injury, and therefore possibly are unable to proliferate properly after injury when compared with their WT counterparts. However, whether this effect reflects an intrinsic property of Gal-9-deficient epithelia, or instead is a consequence of indirect Gal9-mediated effects outside the epithelial compartment, remained unknown. Thus, intestinal crypts were isolated from WT and Gal- $9^{-/-}$mice, and their growth was evaluated ex vivo. Compared with their WT counterparts, Gal- $9^{-1-}$-derived organoids were less complex, with a predominant number of the $\mathrm{Gal}-9^{-1-}$-derived organoids lacking complex three-dimensional structures and instead remaining as nonbudding spheroids (Figure 4, A, C and D). Moreover, when organoids developed into complex three-dimensional structures with buds (eg, enteroids), Gal- $9^{-1-}$-derived organoids had significantly less buds per enteroid (Figure 4B). Taken together, these findings indicate that epithelia that lack proper Gal-9 expression show defective growth ex vivo, suggesting an intrinsic role for Gal-9 in epithelial growth and development.

\section{Gal-9-/- Mice Show Increased Sensitivity to Acute Irradiation-Induced Injury and Have Decreased Wound Closure}

Because the above data indicate that epithelia lacking Gal-9 may possess an intrinsic defect in regeneration, we next challenged Gal- $9^{-1-}$ mice with acute irradiation. Intestinal epithelia are sensitive to high levels of ionizing radiation, and exposure to ionizing radiation is linked to epithelial depletion and stimulation of a robust regenerative response. ${ }^{39} \mathrm{WT}$ and Gal- $9^{-1-}$ mice were challenged with 8 Gy of irradiation, followed by examination of the overall and epithelial-specific response to irradiation injury. Although there were no significant changes in weight loss or acute mortality (data not 

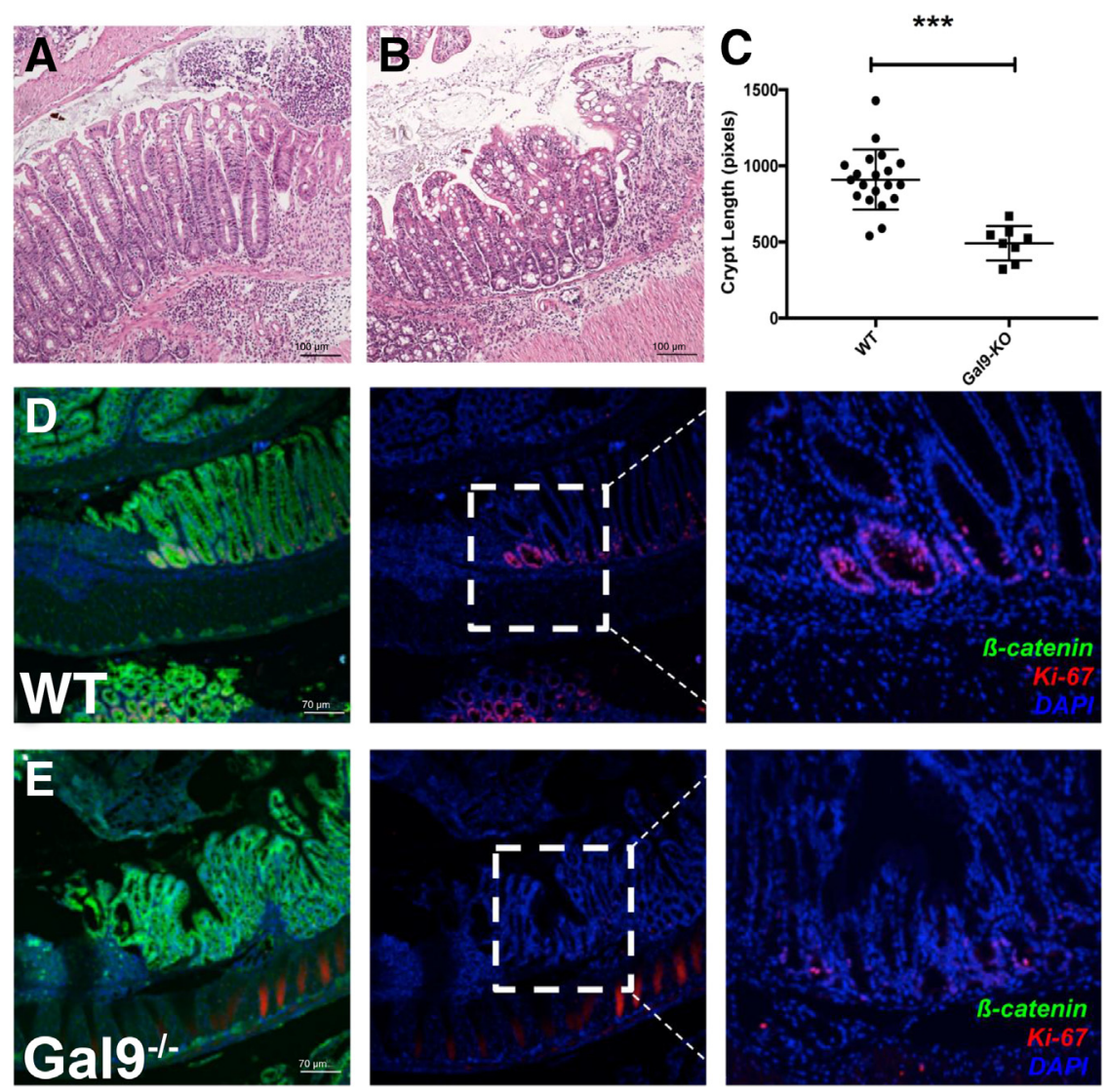

Figure 3 Proliferation responses in regenerating crypts of dextran sodium sulfate (DSS)-treated wild-type (WT) and galectin-9-deficient (Gal-9-/also known as Lgals $^{-1-}$ ) mice. A and B: Representative photomicrographs of crypts adjacent to ulcerated mucosa of DSS-treated mice from WT (A) and Gal- $9^{-1-}$ (B) animals. C: Quantification of crypt length from crypts adjacent to ulcerated mucosa of DSS-treated mice from WT and $\mathrm{Gal}-9^{-1-}$ animals. D and E: Ki-67, DAPI, and ß-catenin immunostaining performed on (D) WT and (E) Gal- $9^{-/-}$animals. Boxed areas indicate ulcerated edges, also known as Lgals $^{-1-}$, which are represented in higher magnification in images to the right. F: Quantification of the Ki-67 proliferation index of crypts adjacent to the ulcerated edge of DSS-treated WT and Gal- $9^{-/-}$mice. ${ }^{*} P<0.005,{ }^{* *} P<0.0005$. Scale bars: $100 \mu \mathrm{m}$ (A and B); $70 \mu \mathrm{m}$ (D and E). K0, knockout.

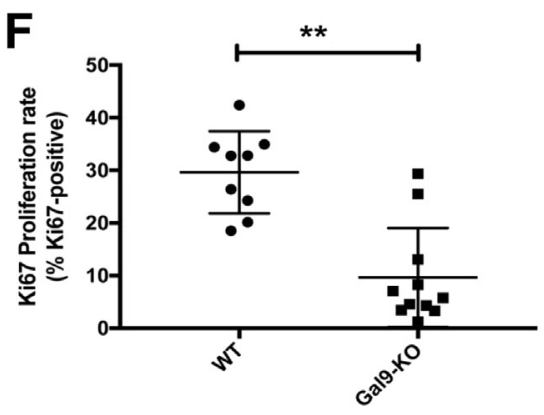

shown), Gal-9 $9^{-l-}$ mice showed marked villous atrophy and crypt depletion 5 days after irradiation compatible with impaired regenerative responses (Figure 5).

Another in vivo model that tests epithelial restitutive responses is wound biopsies. To use this approach, Gal- $9^{-1-}$ animals were challenged with endoscopic wound biopsies followed by examination of wound repair. WT and Gal-9 ${ }^{-/-}$ animals were subjected to miniaturized endoscopy, and biopsy forceps were used to generate defined mucosal wounds in the distal colon of WT and Gal- $9^{-1-}$ mice in a coordinated and highly reproducible fashion. ${ }^{36,37}$ Twenty-four hours after biopsy, endoscopic images were obtained and compared with images obtained 72 hours after biopsy. Compared with WT mice, Gal- $9^{-1-}$ mice showed significantly decreased wound closure (average wound closure of $11 \%$ compared with $35 \%$ in WT mice; $P=0.001$ ), with several of the Gal- $9^{-1-}$ mice showing minimal evidence of wound closure (Figure 6). Taken together, these data show that Gal- $9^{-/-}$animals have defective restitution and wound closure.

\section{Regenerating Crypts in Human Biopsies Show Increased Gal-9 Expression}

Given data indicating that Gal-9 influences proliferating crypts during times of regeneration in murine models of epithelial repair, it was next determined whether Gal-9 expression also is up-regulated in regenerating human epithelia. A mucosal resection containing a healing erosion from a prior biopsy was obtained and stained with an antibody directed against human Gal-9 (Figure 7A). Consistent with data suggesting that Gal-9 acts within epithelia to drive regeneration, expression of Gal-9 was observed within the 

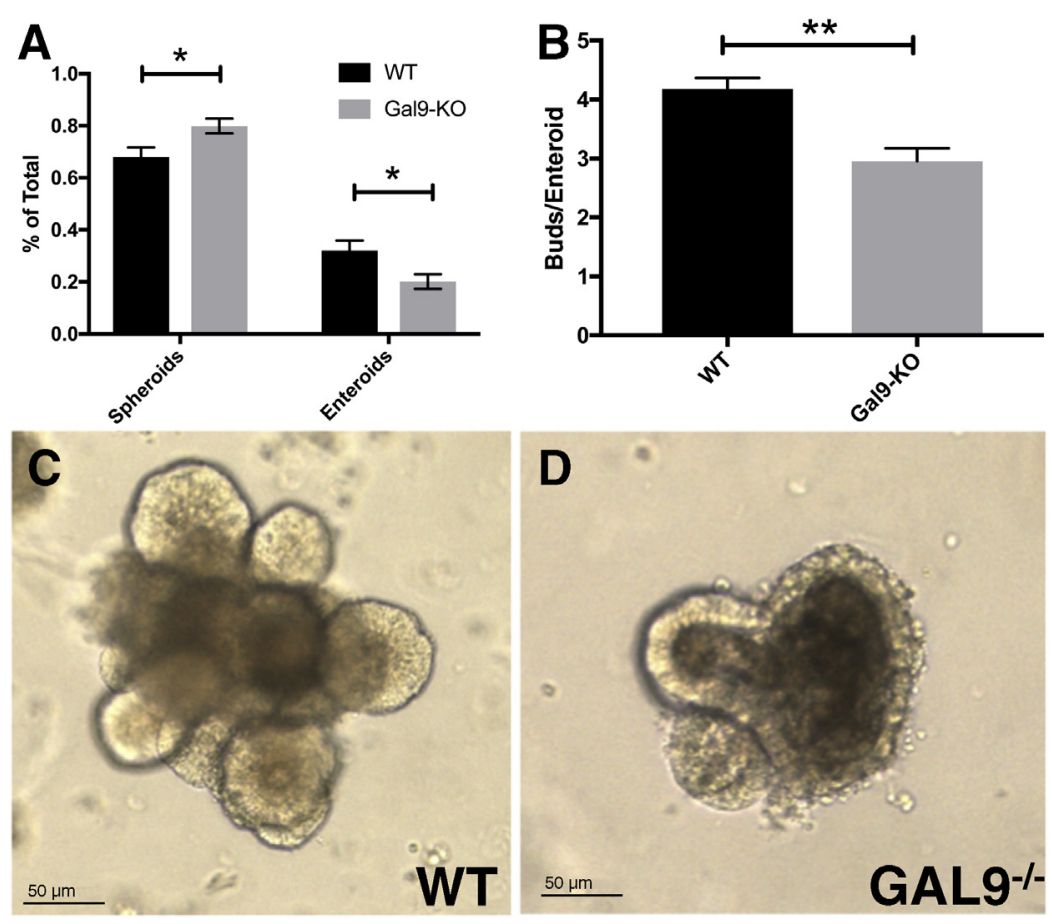

Figure 4 Intestinal organoids derived from wild-type (WT) and galectin-9-deficient (Gal9 ${ }^{-1-}$, also known as Lgals $9^{-1-}$ ) mice. A: The number of spheroids versus enteroids on day 9 preparations. B: The number of buds per enteroid for all visualized enteroids, defined as any budding three-dimensional structure. $\mathbf{C}$ and $\mathbf{D}$ : Representative photomicrographs of 9-day-old intestinal organoids isolated from WT (C) and Gal- $9^{-/}$(D) animals. ${ }^{*} P<0.05,{ }^{*} P<0.005$. Scale bars $=50 \mu \mathrm{m}(\mathbf{C}$ and $\mathbf{D})$. $\mathrm{K} 0$, knockout.

epithelia, which was increased in regenerating crypts adjacent to the wound (Figure 7B).

\section{Discussion}

This study shows that the tandem repeat galectin, Gal-9, impacts epithelial restitution and response across multiple models of intestinal injury. Epithelia devoid of Gal-9 expression show attenuated proliferative responses in the face of regenerative stimuli; these effects appear to be intrinsic to the epithelia because Gal- $9^{-/-}$-derived intestinal epithelial organoids showed defective ex vivo growth in the apparent absence of extrinsic stimuli. These data add to the growing number of studies highlighting galectins as critical regulators of epithelial cell biology.

Gal-9 has a well-described role of regulating aspects of adaptive immunity through interactions with T-cell immunoglobulin and mucin-domain containing-3 (TIM3) and cell differentiation antigen 44 (CD44), where it regulates $\mathrm{T}$ helper type 1 and regulatory $\mathrm{T}$ cell apoptosis and expansion, respectively. ${ }^{40,41}$ In addition, recent studies have highlighted a role
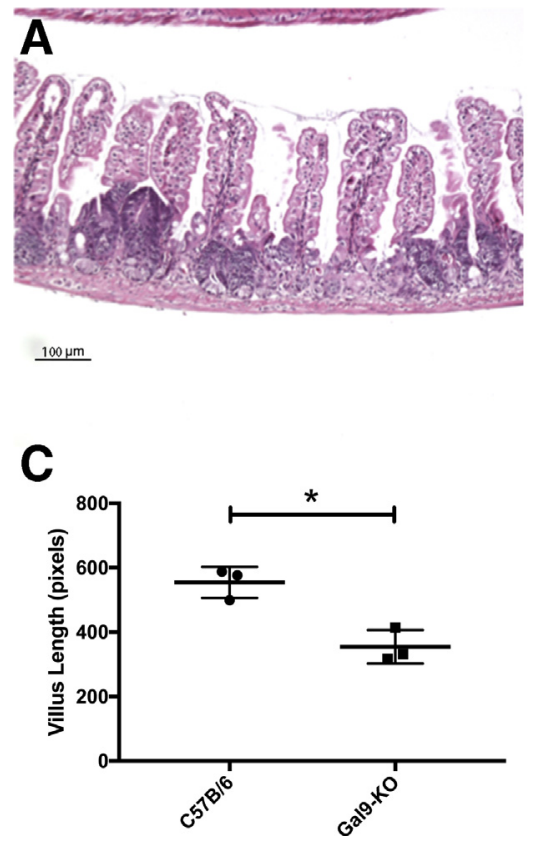
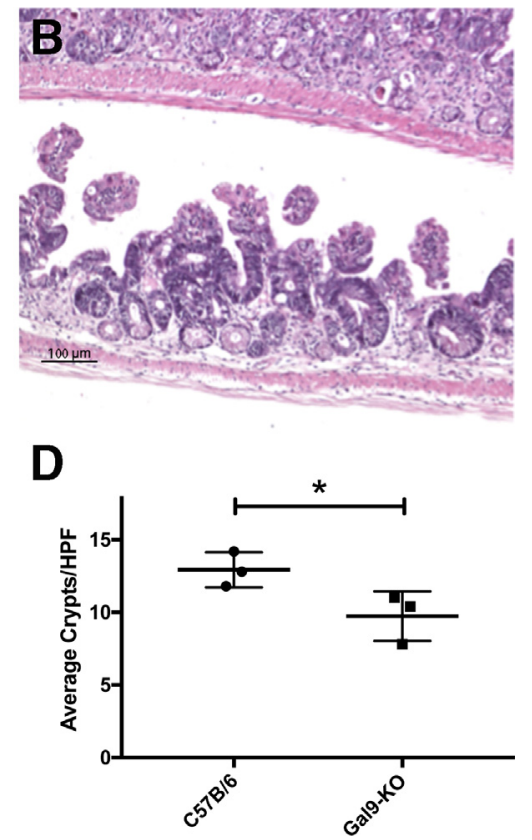

Figure 5 Response to irradiation-induced injury in wild-type (WT) and galectin-9-deficient (Gal9 ${ }^{-/-}$, also known as Lgals $^{-/-}$) mice. A and B: Representative photomicrographs of hematoxylin and eosin staining from midjejunal preparations of mucosa 5 days after irradiation from WT (A) and Gal-9-/- (B) animals. C and D: For each group, the villus length $(\mathbf{C})$ and crypt density $(\mathbf{D})$ is shown. Scale bars $=100 \mu \mathrm{m}(\mathbf{A}$ and $\mathbf{B}) .{ }^{*} P<0.05$. HPF, highpower field; K0, knockout. 

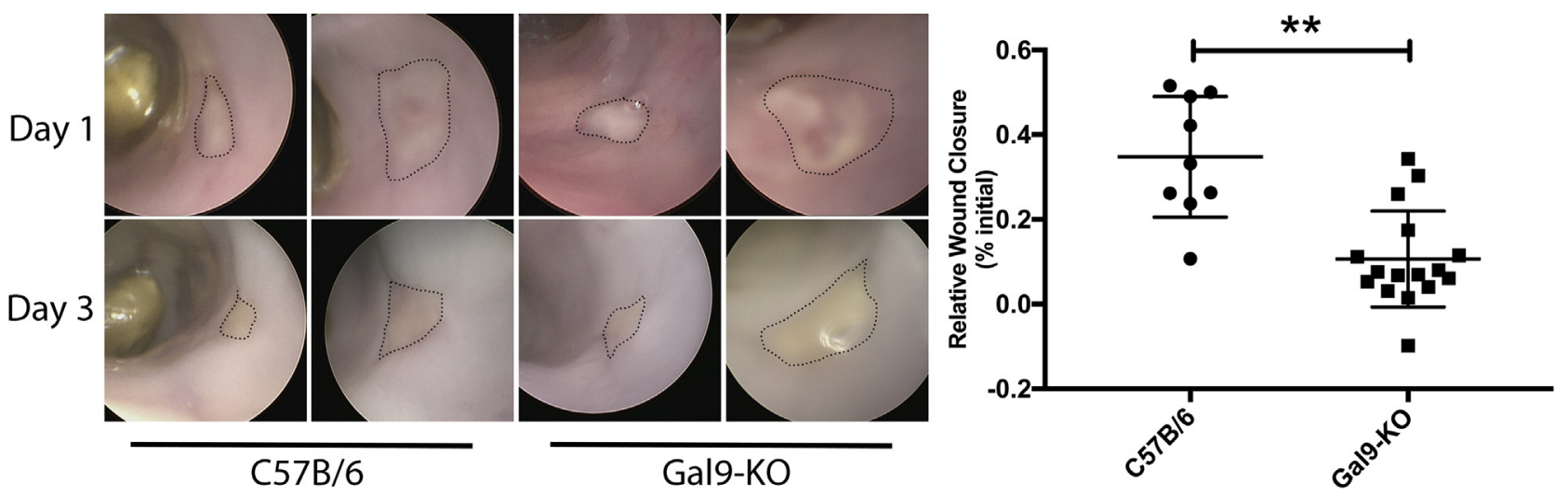

Figure 6 Analysis of response to endoscopic wound biopsy in wild-type (WT) and Gal9-/- (also known as $\mathrm{Lgals}^{-9^{-}}{ }^{-}$) mice. WT and galectin-9-deficient $\left(\right.$ Gal- $\left.-9^{-/}\right)$mice were anesthetized and exposed to endoscopic wounds generated from a forceps biopsy. At 24 hours after the biopsy, wounds generated in WT (top row, left) and Gal9 ${ }^{-/-}$(top row, right) mice were photographed using miniaturized endoscopy and compared with images obtained 72 hours after the biopsy (bottom row left versus right). Dotted lines indicate the wound edge from the biopsy. To the right of the photomicrographs, the percentage of wound closure calculated at 72 hours after the biopsy in WT and Gal- $9^{-1-}$ mice is shown. ${ }^{* *} P<0.005$. K0, knockout.

for endogenous Gal-9 in activating T helper 17 cells and promoting efficient IgA formation. ${ }^{42}$ In allergy models, Gal-9 has a role in regulating interactions between hyaluronan and CD44, thereby augmenting allergic responses. ${ }^{43}$ These regulatory roles in leukocyte biology are likely to influence or may be entirely responsible for certain aspects of the phenotypes observed in the present study; however, because several studies have highlighted that intestinal epithelial cells can express Gal-9 and that Gal-9 expression is dynamic in epithelia, it is likely that Gal-9 exerts additional functions within intestinal epithelial cells. Moreover, in other contexts, endogenous Gal-9 has been shown to act as an alarmin within epithelia, where its expression is induced and functions to regulate restitutive responses. ${ }^{44}$ Thus, Gal-9 may have multiple roles in shaping tissue restitution through acting within or on epithelia (where it is induced), as well as augmenting
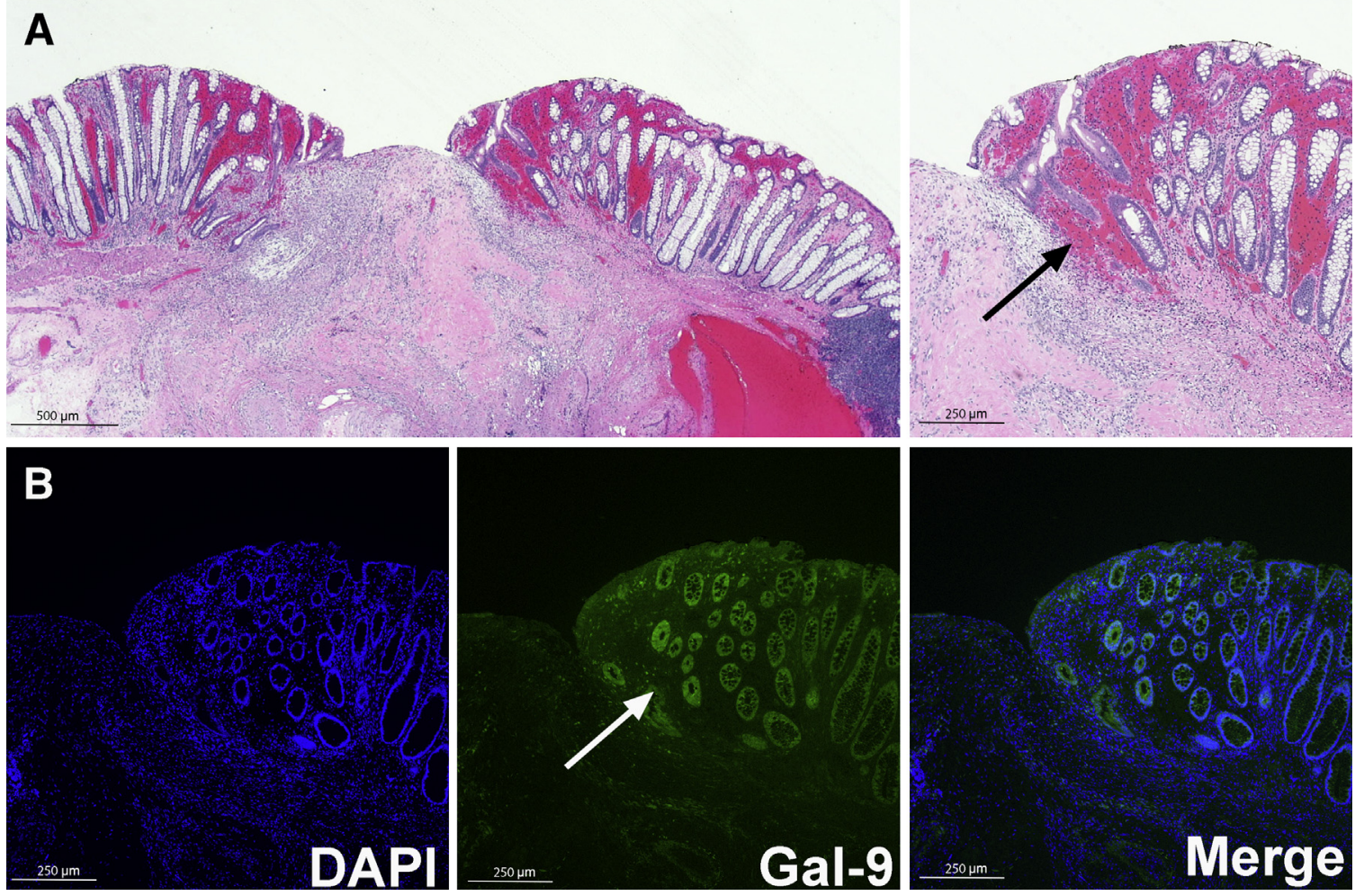

Figure 7 Galectin 9 (Gal-9) expression in human colonic biopsies. A: Hematoxylin and eosin staining of an endoscopic mucosal resection from a patient with a previous procedure resulting in erosion and biopsy-site changes (higher magnification on right, arrow indicates proliferative crypts at eroded edge of wound). B: Serial sections were stained with DAPI and anti-human galectin-9 (Gal-9) antibody; arrow indicates increased Gal-9 expression in proliferating crypts adjacent to biopsy site. Scale bars: $500 \mu \mathrm{m}$ (A, left panel); $250 \mu \mathrm{m}$ (A, right panel, and B). 
mucosal immune responses through favorable regulation of immune function.

How or where Gal-9 exerts its effects on epithelial biology, at present, remains unknown. As mentioned, many studies have highlighted that Gal-9 can act on ligands expressed on the extracellular surface to augment cellular responses. One intriguing possibility within the context of intestinal epithelia is CD44, a known Gal-9 ligand; variant isoforms of CD44 are expressed within the crypts of intestinal epithelia and are known to regulate cell proliferation. ${ }^{45}$ However, a number of studies also have highlighted a role for Gal-9 in augmenting biological functions within the cell. These include studies that have shown that Gal-9 can regulate metabolism through controlling autophagy via direct interactions with AMP-activated protein kinase and AMPK and mitogen-activated protein kinase 7 (TGF- $\beta$ activated kinase 1 , TAK1). ${ }^{46}$ Thus, in addition to engaging extracellular ligands, Gal-9 may act within intracellular compartments to control regenerative responses. Because emerging data have implicated other tandem repeat galectins in the control of microbial populations, an intriguing possibility could be that Gal-9 also exerts effects on the microbiome, which in turn elicits protective effects. ${ }^{47,48}$ Because galectins can function extracellularly and intracellularly, in addition to impacting several different cell types, future studies certainly will be needed to define how galectin-9 influences epithelial biology in vivo.

This data indicate that Gal-9-deficient epithelia have impaired proliferation in the face of regenerative responses. In the context of galectins, effects on the cell cycle are not without precedence. The tandem-repeat galectin Gal-12 was found to show cell-cycle-dependent expression in human Jurkat cells, and overexpression of Gal-12 was found to induce cell-cycle arrest in Hela cells. ${ }^{49}$ Gal-3 has a well-described role in driving breast cancer phenotypes, in part through its role promoting anchorage-independent growth via up-regulation of cyclin D1 in human breast cancer cell lines. ${ }^{50,51}$ In fact, injection of Gal-9 inhibited G1 to G2 progression of human podocytes in a mouse model of diabetic nephropathy. ${ }^{52}$ Aside from these possible specific effects on cell-cycle regulatory proteins, an equally plausible model is that Gal-9 could exert its effects through cell-signaling pathways that impinge on cell-cycle programs. In murine cartilage, Gal-9 was shown to promote osteoblast proliferation through lipid raft stabilization of protooncogene tyrosine-protein kinase Src (c-SRC), which thereby promoted efficient extracellular signal-regulated kinase (ERK) activation and signaling. ${ }^{53}$ In addition, Gal-9 has been shown to control cell metabolism through AMPK and regulate dendritic cells through augmenting p38 and phosphoinositide-3-kinase (Pi3K) signaling. ${ }^{46,54}$ Whether similar effects occur in regenerating epithelia remains unknown. Moreover, whether Gal-9 exerts effects through pathways previously linked to galectindependent regulation, including K-ras, Pi3K, Fos/Jun, and HIF-signaling pathways, remains unknown. ${ }^{55}$ Thus, moving forward, it will be interesting to understand the mechanisms by which Gal-9 exerts these effects on cell proliferation.

\section{References}

1. Camilleri M, Madsen K, Spiller R, Greenwood-Van Meerveld B, Verne GN: Intestinal barrier function in health and gastrointestinal disease. Neurogastroenterol Motil 2012, 24:503-512

2. Oshima T, Miwa H: Gastrointestinal mucosal barrier function and diseases. J Gastroenterol 2016, 51:768-778

3. Khor B, Gardet A, Xavier RJ: Genetics and pathogenesis of inflammatory bowel disease. Nature 2011, 474:307-317

4. Liu TC, Stappenbeck TS: Genetics and pathogenesis of inflammatory bowel disease. Annu Rev Pathol 2016, 11:127-148

5. Viguier M, Advedissian T, Delacour D, Poirier F, Deshayes F: Galectins in epithelial functions. Tissue Barriers 2014, 2:e29103

6. Di Lella S, Sundblad V, Cerliani JP, Guardia CM, Estrin DA, Vasta GR, Rabinovich GA: When galectins recognize glycans: from biochemistry to physiology and back again. Biochemistry 2011, 50:7842-7857

7. Levi G, Teichberg VI: Isolation and physicochemical characterization of electrolectin, a beta-D-galactoside binding lectin from the electric organ of Electrophorus electricus. J Biol Chem 1981, 256:5735-5740

8. Arthur CM, Baruffi MD, Cummings RD, Stowell SR: Evolving mechanistic insights into galectin functions. Methods Mol Biol 2015, 1207:1-35

9. Johannes L, Jacob R, Leffler H: Galectins at a glance. J Cell Sci 2018, 131:jcs208884

10. Robinson BS, Arthur CM, Evavold B, Roback E, Kamili NA, Stowell CS, Vallecillo-Zuniga ML, Van Ry PM, Dias-Baruffi M, Cummings RD, Stowell SR: The sweet-side of leukocytes: galectins as master regulators of neutrophil function. Front Immunol 2019, 10: 1762

11. Kamili NA, Arthur CM, Gerner-Smidt C, Tafesse E, Blenda A, DiasBaruffi M, Stowell SR: Key regulators of galectin-glycan interactions. Proteomics 2016, 16:3111-3125

12. Yang RY, Rabinovich GA, Liu FT: Galectins: structure, function and therapeutic potential. Expert Rev Mol Med 2008, 10:e17

13. Nio J, Kon Y, Iwanaga T: Differential cellular expression of galectin family mRNAs in the epithelial cells of the mouse digestive tract. J Histochem Cytochem 2005, 53:1323-1334

14. Simovic Markovic B, Nikolic A, Gazdic M, Bojic S, Vucicevic L, Kosic M, Mitrovic S, Milosavljevic M, Besra G, Trajkovic V, Arsenijevic N, Lukic ML, Volarevic V: Galectin-3 plays an important pro-inflammatory role in the induction phase of acute colitis by promoting activation of NLRP3 inflammasome and production of IL1beta in macrophages. J Crohns Colitis 2016, 10:593-606

15. Tsai HF, Wu CS, Chen YL, Liao HJ, Chyuan IT, Hsu PN: Galectin-3 suppresses mucosal inflammation and reduces disease severity in experimental colitis. J Mol Med (Berl) 2016, 94:545-556

16. Lippert E, Stieber-Gunckel M, Dunger N, Falk W, Obermeier F, Kunst C: Galectin-3 modulates experimental colitis. Digestion 2015, 92:45-53

17. Houzelstein D, Reyes-Gomez E, Maurer M, Netter P, Higuet D Expression patterns suggest that despite considerable functional redundancy, galectin- 4 and -6 play distinct roles in normal and damaged mouse digestive tract. J Histochem Cytochem 2013, 61:348-361

18. Kim JY, Cho MK, Choi SH, Lee KH, Ahn SC, Kim DH, Yu HS: Inhibition of dextran sulfate sodium (DSS)-induced intestinal inflammation via enhanced IL-10 and TGF-beta production by galectin-9 homologues isolated from intestinal parasites. Mol Biochem Parasitol 2010, 174:53-61

19. Paclik D, Danese S, Berndt U, Wiedenmann B, Dignass A, Sturm A: Galectin-4 controls intestinal inflammation by selective regulation of peripheral and mucosal $\mathrm{T}$ cell apoptosis and cell cycle. PLoS One 2008, 3:e2629

20. Paclik D, Berndt U, Guzy C, Dankof A, Danese S, Holzloehner P, Rosewicz S, Wiedenmann B, Wittig BM, Dignass AU, Sturm A: Galectin-2 induces apoptosis of lamina propria $\mathrm{T}$ lymphocytes and ameliorates acute and chronic experimental colitis in mice. J Mol Med (Berl) 2008, 86:1395-1406 
21. Papa Gobbi R, De Francesco N, Bondar C, Muglia C, Chirdo F, Rumbo M, Rocca A, Toscano MA, Sambuelli A, Rabinovich GA, Docena GH: A galectin-specific signature in the gut delineates Crohn's disease and ulcerative colitis from other human inflammatory intestinal disorders. Biofactors 2016, 42:93-105

22. Jostins L, Ripke S, Weersma RK, Duerr RH, McGovern DP, Hui KY, et al: Host-microbe interactions have shaped the genetic architecture of inflammatory bowel disease. Nature 2012, 491:119-124

23. Arthur CM, Patel SR, Smith NH, Bennett A, Kamili NA, Mener A, Gerner-Smidt C, Sullivan HC, Hale JS, Wieland A, Youngblood B, Zimring JC, Hendrickson JE, Stowell SR: Antigen density dictates immune responsiveness following red blood cell transfusion. J Immunol 2017, 198:2671-2680

24. Mener A, Arthur CM, Patel SR, Liu J, Hendrickson JE, Stowell SR: Complement component 3 negatively regulates antibody response by modulation of red blood cell antigen. Front Immunol 2018, 9:676

25. Mener A, Patel SR, Arthur CM, Chonat S, Wieland A, Santhanakrishnan M, Liu J, Maier CL, Jajosky RP, Girard-Pierce K, Bennett A, Zerra PE, Smith NH, Hendrickson JE, Stowell SR: Complement serves as a switch between $\mathrm{CD} 4+\mathrm{T}$ cell-independent and -dependent RBC antibody responses. JCI Insight 2018, 3: e121631

26. Kim JJ, Shajib MS, Manocha MM, Khan WI: Investigating intestinal inflammation in DSS-induced model of IBD. J Vis Exp 2012, 60: 3678

27. McDonald JT, Kim K, Norris AJ, Vlashi E, Phillips TM, Lagadec C, Della Donna L, Ratikan J, Szelag H, Hlatky L, McBride WH: Ionizing radiation activates the Nrf2 antioxidant response. Cancer Res 2010, 70:8886-8895

28. Reddy VK, Short SP, Barrett CW, Mittal MK, Keating CE, Thompson JJ, Harris EI, Revetta F, Bader DM, Brand T, Washington MK, Williams CS: BVES regulates intestinal stem cell programs and intestinal crypt viability after radiation. Stem Cells 2016, 34:1626-1636

29. Erben U, Loddenkemper C, Doerfel K, Spieckermann S, Haller D, Heimesaat MM, Zeitz M, Siegmund B, Kuhl AA: A guide to histomorphological evaluation of intestinal inflammation in mouse models. Int J Clin Exp Pathol 2014, 7:4557-4576

30. Schindelin J, Arganda-Carreras I, Frise E, Kaynig V, Longair M, Pietzsch T, Preibisch S, Rueden C, Saalfeld S, Schmid B, Tinevez J-Y, White DJ, Hartenstein V, Eliceiri K, Tomancak P, Cardona A: Fiji: an open-source platform for biological-image analysis. Nat Methods 2012, 9:676-682

31. Cerri DG, Arthur CM, Rodrigues LC, Fermino ML, Rocha LB, Stowell SR, Baruffi MD: Examination of galectin localization using confocal microscopy. Methods Mol Biol 2015, 1207:343-354

32. Schmittgen TD, Livak KJ: Analyzing real-time PCR data by the comparative C(T) method. Nat Protoc 2008, 3:1101-1108

33. Sato T, Vries RG, Snippert HJ, van de Wetering M, Barker N, Stange DE, van Es JH, Abo A, Kujala P, Peters PJ, Clevers H: Single Lgr5 stem cells build crypt-villus structures in vitro without a mesenchymal niche. Nature 2009, 459:262-265

34. Mahe MM, Aihara E, Schumacher MA, Zavros Y, Montrose MH, Helmrath MA, Sato T, Shroyer NF: Establishment of gastrointestinal epithelial organoids. Curr Protoc Mouse Biol 2013, 3:217-240

35. Chen G, Bei B, Feng Y, Li X, Jiang Z, Si JY, Qing DG, Zhang J, Li N: Glycyrrhetinic acid maintains intestinal homeostasis via HuR. Front Pharmacol 2019, 10:535

36. Alam A, Leoni G, Wentworth CC, Kwal JM, Wu H, Ardita CS, Swanson PA, Lambeth JD, Jones RM, Nusrat A, Neish AS: Redox signaling regulates commensal-mediated mucosal homeostasis and restitution and requires formyl peptide receptor 1. Mucosal Immunol 2014, 7:645-655

37. Seno H, Miyoshi H, Brown SL, Geske MJ, Colonna M, Stappenbeck TS: Efficient colonic mucosal wound repair requires Trem2 signaling. Proc Natl Acad Sci U S A 2009, 106:256-261
38. Scholzen T, Gerdes J: The Ki-67 protein: from the known and the unknown. J Cell Physiol 2000, 182:311-322

39. Potten CS: Radiation, the ideal cytotoxic agent for studying the cell biology of tissues such as the small intestine. Radiat Res 2004, 161: $123-136$

40. Wu C, Thalhamer T, Franca RF, Xiao S, Wang C, Hotta C, Zhu C, Hirashima M, Anderson AC, Kuchroo VK: Galectin-9-CD44 interaction enhances stability and function of adaptive regulatory $\mathrm{T}$ cells. Immunity 2014, 41:270-282

41. Zhu C, Anderson AC, Schubart A, Xiong H, Imitola J, Khoury SJ, Zheng XX, Strom TB, Kuchroo VK: The Tim-3 ligand galectin-9 negatively regulates $\mathrm{T}$ helper type 1 immunity. Nat Immunol 2005, 6:1245-1252

42. Liang CC, Li CS, Weng IC, Chen HY, Lu HH, Huang CC, Liu FT: Galectin-9 is critical for mucosal adaptive immunity through the T helper 17-IgA axis. Am J Pathol 2018, 188:1225-1235

43. Katoh S, Ishii N, Nobumoto A, Takeshita K, Dai SY, Shinonaga R, Niki T, Nishi N, Tominaga A, Yamauchi A, Hirashima M: Galectin-9 inhibits CD44-hyaluronan interaction and suppresses a murine model of allergic asthma. Am J Respir Crit Care Med 2007, 176:27-35

44. Hirao H, Uchida Y, Kadono K, Tanaka H, Niki T, Yamauchi A, Hata K, Watanabe T, Terajima H, Uemoto S: The protective function of galectin-9 in liver ischemia and reperfusion injury in mice. Liver Transpl 2015, 21:969-981

45. Zeilstra J, Joosten SP, van Andel H, Tolg C, Berns A, Snoek M, van de Wetering M, Spaargaren M, Clevers H, Pals ST: Stem cell CD44v isoforms promote intestinal cancer formation in $\mathrm{Apc}(\mathrm{min})$ mice downstream of Wnt signaling. Oncogene 2014, 33:665-670

46. Jia J, Abudu YP, Claude-Taupin A, Gu Y, Kumar S, Choi SW, Peters R, Mudd MH, Allers L, Salemi M, Phinney B, Johansen T, Deretic V: Galectins control MTOR and AMPK in response to lysosomal damage to induce autophagy. Autophagy 2019, 15:169-171

47. Arthur CM, Patel SR, Mener A, Kamili NA, Fasano RM, Meyer E, Winkler AM, Sola-Visner M, Josephson CD, Stowell SR: Innate immunity against molecular mimicry: examining galectin-mediated antimicrobial activity. Bioessays 2015, 37:1327-1337

48. Stowell SR, Arthur CM, Dias-Baruffi M, Rodrigues LC, Gourdine JP, Heimburg-Molinaro J, Ju T, Molinaro RJ, Rivera-Marrero C, Xia B, Smith DF, Cummings RD: Innate immune lectins kill bacteria expressing blood group antigen. Nat Med 2010, 16:295-301

49. Yang RY, Hsu DK, Yu L, Ni J, Liu FT: Cell cycle regulation by galectin-12, a new member of the galectin superfamily. J Biol Chem 2001, 276:20252-20260

50. Yoshii T, Fukumori T, Honjo Y, Inohara H, Kim HR, Raz A: Galectin-3 phosphorylation is required for its anti-apoptotic function and cell cycle arrest. J Biol Chem 2002, 277:6852-6857

51. Kim HR, Lin HM, Biliran H, Raz A: Cell cycle arrest and inhibition of anoikis by galectin-3 in human breast epithelial cells. Cancer Res 1999, 59:4148-4154

52. Baba M, Wada J, Eguchi J, Hashimoto I, Okada T, Yasuhara A, Shikata K, Kanwar YS, Makino H: Galectin-9 inhibits glomerular hypertrophy in $\mathrm{db} / \mathrm{db}$ diabetic mice via cell-cycle-dependent mechanisms. J Am Soc Nephrol 2005, 16:3222-3234

53. Tanikawa R, Tanikawa T, Okada Y, Nakano K, Hirashima M, Yamauchi A, Hosokawa R, Tanaka Y: Interaction of galectin-9 with lipid rafts induces osteoblast proliferation through the c-Src/ERK signaling pathway. J Bone Miner Res 2008, 23:278-286

54. de Kivit S, Kostadinova AI, Kerperien J, Ayechu Muruzabal V, Morgan ME, Knippels LMJ, Kraneveld AD, Garssen J, Willemsen LEM: Galectin-9 produced by intestinal epithelial cells enhances aldehyde dehydrogenase activity in dendritic cells in a PI3K- and p38-dependent manner. J Innate Immun 2017, 9:609-620

55. Laderach DJ, Compagno D, Toscano MA, Croci DO, Dergan-Dylon S, Salatino M, Rabinovich GA: Dissecting the signal transduction pathways triggered by galectin-glycan interactions in physiological and pathological settings. IUBMB Life 2010, 62:1-13 